\title{
Is serodiagnosis of herpes simplex encephalitis safe?
}

\author{
J.G. Coghlan, C. Monaghan, P.A. Carney and J.H. Moran \\ Department of Neurology and Department of Psychiatry, Regional Hospital, Galway, Irish Republic
}

\begin{abstract}
Summary: We present a case of probable tuberculous meningitis in which serological changes 'diagnostic' of herpes simplex encephalitis were found. Evidence is provided that the serological changes in this case represent a true false positive, and that reliance on clinical plus serological criteria to diagnose herpes simplex encephalitis could result in failure to diagnose and treat tuberculous meningitis.
\end{abstract}

\section{Introduction}

Sensitive techniques for the sero-diagnosis of herpes simplex encephalitis (HSE) have been developed and some authorities no longer require brain biopsy for the diagnosis of HSE. ${ }^{1}$ Sero-diagnosis is known to give false positive results in $12-19 \%$ of cases, but it has been suggested that these false positives occur only in conditions which are themselves readily diagnosed and therefore are not a cause of confusion. ${ }^{2}$ Tuberculous meningitis (TBM) is a cause of non-specific polyclonal B-cell activation, and has been shown to produce non-specific antibody activity against herpes simplex ${ }^{3}$ but may be extremely difficult to diagnose. We believe that this patient with a classical presentation of TBM and serological changes 'diagnostic' of HSE shows that sero-diagnosis of HSE is not yet safe.

\section{Case report}

Two weeks before admission a 42 year old woman had two grand mal seizures and subsequently had become increasingly withdrawn, disoriented and was refusing to eat. As she refused hospital admission she was certified and admitted under the care of the psychiatric department.

Physical examination revealed a pyrexia of $37.5^{\circ} \mathrm{C}$, a mild global dysphasia and evidence of recent weight loss. Mental state examination revealed that she was highly distractible, disoriented in time, person and place and was confabulating.

Initial investigations included full blood count, erythrocyte sedimentation rate, blood glucose, urea and electrolytes, serum calcium, liver function tests, sputum and urine cultures for acid-fast bacteria, serology for brucella, syphilis, cytomegalovirus, and

Correspondence: J.G. Coghlan M.B., M.R.C.P.I. Meath Hospital, Heytesbury Street, Dublin 8, Irish Republic Accepted: 30 April 1987 toxoplasmosis, all of which were normal. Antinuclear, antimitochondrial and antismooth muscle antibody titres were less than 1:20. In addition thyroid function tests, serum lead levels, skull, chest and abdominal radiograph electroencephalograph and isotope brain scan were normal.

The pyrexia noted on admission settled within 24 hours and did not recur. However her dysphasia worsened over the next two weeks, subsequently she became irritable and she exhibited increasing suspicion of others suggestive of paranoid delusions. By the fifth week of her illness she was aphasic and her behaviour suggested that she had a profound psychosis.

Lumbar puncture initially revealed an increase in lymphocytes $\left(30 \times 10^{9} / 1\right)$ and protein $(2.4 \mathrm{~g} / 1)$ with a normal glucose suggestive of an encephalitic process. Repeated electroencephalograms at no time revealed evidence of encephalitis, and computed tomographic (CT) scan was normal. However repeat lumbar puncture on the fifth week of her illness revealed a reduced glucose $(2.2 \mathrm{mmol} / \mathrm{l})$, increased protein $(3.9 \mathrm{~g} / \mathrm{l})$, and increased lymphocytes $\left(45 \times 10^{9} / 1\right)$, strongly suggestive of TBM. The Mantoux test at $100 \mathrm{TU}$ was negative.

Therapy with isoniazid and rifampicin was therefore commenced. The patient responded rapidly to this therapy and she made an almost complete recovery. After 4 weeks of treatment her paranoia and disorientation had cleared, her dysphasia after 7 weeks and her distractibility after 11 weeks. Three months after commencing therapy intellectual function tests still reveal a mild general impairment of memory but are otherwise normal.

Cultures of the cerebrospinal fluid (CSF) for bacteria, fungi and mycobacteria were negative on all occasions. An eight fold rise in CSF anti-herpes simplex titres (complement fixation test) was noted between the fifth and sixth week of illness, and serum: 
CSF ratios were less then 10:1. Further convalescent measurements revealed a four fold drop in CSF antiHS titres with convalescence.

The antibody index ${ }^{4}$ was used to demonstrate integrity of the blood brain barrier, showing that intrathecal production of anti-HS antibodies was occurring. On the eighth week of her illness serum anti-HS titres were $1: 640$, CSF titres were $1: 80$, serum albumen was $3,760 \mathrm{mg} / \mathrm{dl}$ and CSF albumen was $42.9 \mathrm{mg} / \mathrm{dl}$, yielding a considerably elevated antibody index of 10.9 (normal 0-1.46).

\section{Discussion}

The diagnosis of TBM may be extremely difficult as no organism is isolated in up to $30 \%$ of cases and the Mantoux test is negative in $70 \%$ of cases. ${ }^{5}$ Thus it is frequently necessary to make this diagnosis clinically as in this case. Both the nature and the course of this patient's illness were typical of the neuropsychiatric presentation of TBM. ${ }^{6}$ She developed typical CSF changes on the fifth week of her illness and her response to therapy followed the usual course of the treated disease. ${ }^{\circ}$ She therefore fulfils Sada's criteria for the diagnosis of probable TBM. ${ }^{?}$

HSE usually presents as an acute severe organic brain syndrome often with progression to coma within a week of onset; subacute, mild and other atypical presentations have however been described. ${ }^{8}$ Brain biopsy is the accepted method of diagnosing HSE as it

\section{References}

1. Skoldenberg, B., Kalimo, K., Carlstrom, A., Forsgren, M. \& Haconen, P. Herpes simplex encephalitis - a serological follow-up study. Acta Neurol Scand 1981, 63: 273-285.

2. Nahmais, A.J., Whitney, R.J., Vistine, A.N., Takei, Y.\& Alford, C.A.M. Herpes simplex virus encephalitis: laboratory evaluations and their diagnostic significance. $J$ Infect Dis 1982, 145: 829-836.

3. Kinnman, J., Link, H. \& Fryden, A. Characterisation of antibody activity in oligoclonal immunoglobulin G, synthesised within the central nervous system in a patient with tuberculous meningitis. J Clin Microbiol 1981, 13: 30-35.

4. Klapper, P.E., Laing, I. \& Longson, M. Rapid non invasive diagnosis of herpes encephalitis. Lancet 1981, ii: 607-608.

5. Traub, M., Colchester, A.C.F., Kingsley, D.P.E. \& Swa- is both specific and sensitive and causes very little $\frac{\varrho}{3}$

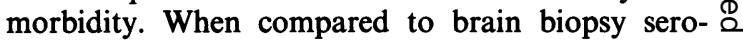
diagnosis is non-specific, as diagnostic titres (serum: $\leftarrow$ CSF ratio $<20 / 1$ ) occur $19 \%$ of the time, ${ }^{2}$ false $\overrightarrow{\vec{A}}$ positives were, however, felt to occur only in conditions which were themselves readily diagnosed (e.g. other encephalitides and multiple sclerosis). Serodiagnosis was mainly criticized initially for its lack of sensitivity; however, the development of radio im- $\varrho$ munoassay techniques has circumvented this problem in and Skoldenberg suggests that a combination of $\vec{\circ}$ clinical and serological findings consistent with HSE is sufficient to make a diagnosis. ${ }^{1}$

Despite serological changes diagnostic of HSE it would be unreasonable to suggest HSE as a diagnosis in this case since an alternative diagnosis is available, $a$. subacute course is rare in HSE and hypoglychorroea $\omega$ as found in this case occurs in only $1 \%$ of cases of HSE. ${ }^{9}$

Considering the diseases known to give false positive results it is apparent that these all cause nonspecific polyclonal B-cell activation. TBM is also known to cause this and further, evidence of anti-HS antibody activity has previously been found in the CSF of a patient with TBM. ${ }^{3}$ We propose that this case confirms that TBM can cause serological changes 'diagnostic' of HSE, as previously suspected and that $\frac{}{-}$ reliance on clinical and serological criteria to diagnos $\overrightarrow{0}$ HSE could lead to disaster for patients with an acute presentation of TBM.

sh, M. Tuberculosis of the central nervous system. $Q J \overline{\overline{\mathrm{O}}}$ Med 1984, 209: 81-100.

6. Lishman, W.A. In The Physiological Consequences of Cerebral Disorders, Vol. 2. Blackwell Scientific Publications, London, 1978, p438.

7. Sada, E., Ruiz-Palacios, G.M., Lopez-Vidal, Y. \& Ponce $\underline{3}$ de Leon, $S$. Detection of mycobacterial antigens in cerebrospinal fluid of patients with tuberculous menin- 3 . gitis by enzyme linked immunosorbent assay. Lancet $\dot{\rho}$ 1983, ii: $651-652$.

8. Oomen, K.J., Johnson, P.C. \& Ray, C.G. Herpes simplex Type II virus encephalitis presenting as psychosis. Am J Med 1982, 73: 445-448.

9. Whitney, R.J., Soong, S., Linneman, C., Liu, C., Paz- 을 in, G. \& Alford, C.A. Herpes simplex encephalitis: clin- N ical assessment. JAMA 1982, 247: 317-320. 